\title{
COMBINING STELLAR EVOLUTION AND
}

\section{STELLAR DYNAMICS}

\author{
PETER P. EGGLETON \\ Institute of Astronomy \\ Cambridge, $U K$
}

\section{Introduction}

There seem to me to be four approaches to the problem of computing the evolution of star clusters. Firstly, one might assume that our knowledge of the evolution of stars can be condensed into a subroutine that can be added to an $\mathrm{N}$-body code. This subroutine would mainly have to give the radius and the time-dependent mass of a star as a function of its initial mass and its age. Secondly, standing this on its head, one might assume that our knowledge of $\mathrm{N}$-body evolution can be condensed into a subroutine that can be added to a stellar evolution code. This subroutine would determine, probably in a Monte-Carlo fashion, whether the star had picked up, or lost, a binary companion, or whether the orbit of its companion was significantly changed; the probabilities would be determined by simple analytic approximations to the time-dependent distribution functions of stars (and binaries) of different masses and ages, and by interaction cross-sections as functions of density and 'temperature'. Thirdly, if the computing power is available, one might more simply unite an N-body code with a Stellar Evolution (SE) code, and follow both the dynamics and the internal evolution simultaneously. Fourthly, we might hope at some stage to put together simple analytic approximations both from N-body and from SE studies, to develop a unified simple model. I venture to say that it is only the last stage, if it is attainable, that would entitle us to say that we 'understand' the evolution of stellar clusters. 'Understanding', I think, means that we can extract some essential wisdom from large numerical simulations, and apply it on the back of the proverbial envelope.

All of these approaches have problems. One that is common to all four is that the SE of interactive binaries is nothing like as well-determined as the $\mathrm{SE}$ of single stars, or of stars that are 'effectvely single', i.e. in 
binaries with periods $\gtrsim 10^{4} \mathrm{~d}$. I shall return to this later; but, putting it aside for the moment in the hope that interactive binary SE can somehow be fudged, then the first approach is probably the easiest to implement. Aarseth (1995) has incorporated into his N-body code a subroutine based on the simple interpolation formulae of Eggleton, Fitchett \& Tout (1989) for the evolution from ZAMS to neutron star or white dwarf of Pop I stars. We (Drs C. A. Tout, Zh. Han and myself) are currently trying to upgrade these formulae to incorporate more recent data on opacities (Rogers \& Iglesias 1992, Alexander \& Ferguson 1994) and the equation of state and other physical input (Pols et al. 1995). We also intend to generalise our formulae for a range of metallicities from Pop I to a fairly extreme Pop II.

The second approach does not yet appear to be feasible. One can hope that as N-body simulations approach $\sim 10^{5}$ bodies some systematic results will emerge regarding the way in which particles in different mass bins distribute themselves in phase space, as a function of time. However, since core collapse phases, and subsequent reexpansion phases due to the formation of central binaries, maybe be somewhat chaotic if the number of particles is large enough, it is not clear that simple interpolation formulae will ever describe such processes reliably. But I should emphasise that SE may also have chaotic aspects. It is not clear that mass loss is such a straightforward process that the mass of a star can be reliably predicted at later times from its mass at age zero; and certain aspects of interactive binary SE, especially those which depend on mass loss (ML) or angular momentum loss (AML), may also show chaotic behaviour.

The third approach, involving brute force, may well be feasible in a decade or two. It would need an SE code that is rather more reliable than any that exists now. My own code, developed over the last 25 years, is now (Han, Podsiadlowski \& Eggleton 1994; Pols et al. 1995) almost reliable enough to follow a star, without manual intervention, from the ZAMS to carbon burning, except that the helium flash in low-mass stars is still an obstacle. It is not impossible, though, that we can fudge our way through that, and so make it fully automatic. On a modern work-station it takes about 2 hours to compute the entire evolution of a star, provided that for stars which have a helium flash ( $\$ 2.3 M_{\odot}$ for Pop I) we ignore the time it takes at present to restart the star manually on the horizontal branch. My SE code can also take care of certain kinds of Roche Lobe overflow (RLOF) in interacting binaries, but it cannot yet deal automatically with contact binary evolution - nor can anyone else's.

In the fourth approach, which I hope will take place in the fullness of time, we might get away with something like the 'gas-dynamical' models of Hachisu et al. (1978), Lynden-Bell \& Eggleton (1980) and Bettweiser \& Sugimoto (1984), in which the whole cluster is treated like a gas sphere 
with a 'temperature' and 'pressure'. The analogue of 'composition' in an SE code would be the abundance fractions, as functions of position, for different mass bins, and for binaries and triples. These different species would interact through the analogue of nuclear reactions, for which the rates would have to be estimated. If, as a last step, one is able to extract from such calculations a few simple insights, expressible as analytic approximations, one might finally feel that cluster evolution has been 'understood' - although I dare say not everyone would agree.

There are at least two ways in which one might transfer information on stellar evolution to an N-body code. One might try to express the SE results in the form of interpolation formulae (Eggleton, Fitchett \& Tout 1989): as simple formulae as possible, though one should probably aim for an accuracy better than $\sim 10 \%$. Alternatively, one can provide the information in tables (e.g. Schaller et al. 1992). Perhaps 30 different masses between 0.1 and $100 M_{\odot}$, each tabulated at about 50 well-chosen evolutionary stages, might be adequate for an accuracy of between $1 \%$ and $10 \%$. There is not much point in aiming for higher accuracy, since for some masses (particularly high masses) and some evolutionary stages there is much bigger disagreement between theoretical and observed models. Even where disagreement is not obvious, one should not suppose that the theoretical models are good. Although several main-sequence stars, i.e. those in eclipsing double-lined spectroscopic binaries, have masses, radii and luminosities measured to $\sim 1 \%$ (Popper 1980, Andersen 1991), very few evolved stars, such as red giants and supergiants, Wolf-Rayet stars, hot subdwarfs and white dwarfs, have masses, radii and luminosities which are known to $10 \%$. Even in rare cases where something like this accuracy is achieved for such stars, it is normally uncertain what the initial masses were, and so it is not clear that there is agreement between observation and theory.

Some major sources of uncertainty in SE calculations are:-

(a) Mass loss (ML). In low-mass ( $\left.\$ 2.3 M_{\odot}\right)$ and intermediate-mass $(\sim 2.3-$ $\left.8 M_{\odot}\right)$ stars there are probably two main types of ML. These are:

(a1) A weak, fairly steady wind which increases during the first giantbranch (FGB) and asymptotic giant-branch (AGB) stages. This wind may be driven by magnetohydrodynamic energy, as in the solar wind, although at high FGB and AGB luminosities it may be that magnetic energy is less important, and turbulent hydrodynamic energy in the surface convection zone may be the prime cause of the wind. Judge \& Stencel (1991) found an empirical relation of the form $-\dot{m} \sim 10^{-13.6} / g^{1.43} M_{\odot} / \mathrm{yr}$, with gravity $g$ in solar units. There is quite considerable scatter, however, and it is by no means clear that this can all be attributed to measurement uncertainties. Even near the top of the AGB, however, where $g \sim 10^{-5} g_{\odot}$, this ML rate is barely comparable to the nuclear timescale. 
(a2) A 'superwind', which removes the remaining envelope at the top of the AGB rather rapidly, forming a planetary nebula with a hot subdwarf (or Wolf-Rayet-like) nucleus. This superwind may be ultimately due to the fact that thanks to progressive hydrogen recombination in the outer layers the total binding energy of the AGB envelope changes from positive to negative (Paczyński \& Ziółkowski 1968, Han et al. 1994). The latter authors, by computing the binding energy of the envelope of a star as it climbs the AGB, and assuming that the envelope is lost at the stage when this energy passes through zero, found a relation between the initial mass (in the range $0.8-7 M_{\odot}$ ) and the final (i.e. WD) mass of a star. This relation, which I would emphasise has no free parameters in it to be 'tuned', was in fairly good agreement with the semi-empirical relation of Weidemann \& Koester (1983) between the initial and final masses of stars of stars in galactic clusters. But we should note that the relation of Weidemann \& Koester (1983) has considerable scatter in it, some of which might be real; it is by no means established that the mass of a WD remnant is tightly correleted with initial mass for a given metllicity, and there may well be a chaotic aspect to the ML process which could lead to a real spread.

For massive stars $\left(~ z 30 M_{\odot}\right)$ there may be four stages of ML:

(a3) A weak but growing wind, to some extent as in (a1) but with a much higher velocity $(\sim 1500 \mathrm{~km} / \mathrm{s}$ as against $30-300 \mathrm{~km} / \mathrm{s})$. Radiation pressure is probably what accelerates the wind to the high speed observed, but the wind may be energised in the first place by MHD processes and by rapid rotation. Conti (1982) plotted ML rate against luminosity, and this relation can be fitted by $-\dot{m} \sim 10^{-16.7} L^{1.9} M_{\odot} / \mathrm{yr}$, with luminosity $L$ in solar units. Once again there is considerable scatter. Though relatively stronger than (a1) at early evolutionary stages, this wind probably also does not affect a star strongly on nuclear timescales.

(a4) A P Cyg wind, which is very copious as well as fast, and which presumably can remove $20-80 \%$ of the star's mass on substantially less than a nuclear timescale. This requires $-\dot{m} \gtrsim 10^{-4} M_{\odot} /$ yr. The $\mathrm{P}$ Cyg wind may start when the star reaches a gently sloping line in the HRD - the HumphreysDavidson (1979) limit, Figure 1 - and may be due to some inherent instability perhaps related to the fact that the interiors of such stars are very near the Eddington limit. It appears to prevent the star from becoming a red supergiant, although not from becoming a yellow supergiant at least at the lower masses $\left(\sim 30-40 M_{\odot}\right)$.

(a5) A Wolf-Rayet wind, which may be a continuation of the P Cyg wind at slightly lower intensity $\left(-\dot{m} \sim 10^{-4.4} M_{\odot} / \mathrm{yr} ;\right.$ Willis 1985$)$ once the star has been stripped nearly or entirely to its small, very hot helium core. This ML rate may be roughly comparable to the (helium burning) nuclear timescale of the star. 

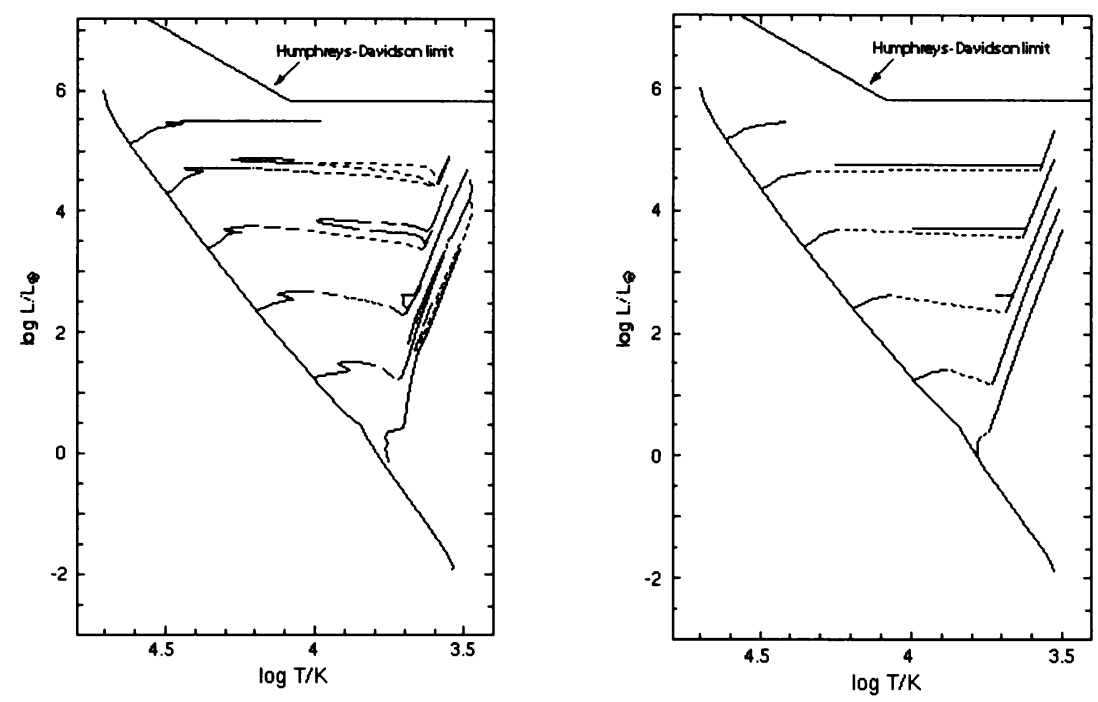

Figure 1. Comparison, in a theoretical Hertzsprung-Russell diagram, of some evolutionary tracks computed (left) directly with a stellar evolution code, and (right) with the simple interpolation formulae given by Eggleton, Fitchett \& Tout (1989). Above the Humphreys-Davidson (1979) limit, virtually no stars are found in the Galaxy, presumably because of strong winds.

(a6) An SN ejection of the remaining envelope, virtually instantaneously. Stars in the mass range $\sim 8-30 M_{\odot}$ probably do not go through the P Cyg phase (a4) or the WR phase (a5). Perhaps they start with (a3) and finish with (a6), passing through a combination of (a2) and (a4), but at a less intense level than in lower and higher mass stars respectively, on the way. It is far from clear how much mass they lose in this intermediate phase.

(b) Semiconvective mixing. Stars of virtually all masses require semiconvective mixing at some point in their evolution. Semiconvection is a difficult physical process, well discussed by Spruit (1992). There is no clear numerical model for this, although in my opinion a model which assumes that mixing always occurs at a rate that keeps the temperature gradient very close to the marginal adiabatic rate (Schwarzschild \& Härm 1958), except in the surface layers, is the most likely to be realistic. It is quite probable that real semiconvection is a somewhat chaotic process. 2-D hydrodynamic, or better still 3-D models, may cast some light on this, but such modeling is in its infancy (Merryfield 1995).

(c) 'Convective overshooting'. To improve agreement between theory and observation of some of the most evolved stars within the MS band (Andersen 1991), it is helpful to assume that convective cores in the MS band 
are larger than is indicated by the standard Schwarzschild criterion. This is also the conclusion of a study of red giants and supergiants in $\zeta$ Aur binaries, i.e. those (rather few) binaries from which the most accurate masses, radii and luminosities of highly evolved stars are obtainable (Schröder 1995, private communication). It is also suggested by attempts to fit the HRDs of young clusters (Meynet, Mermilliod \& Maeder 1993; Pols 1995, private communication). The problem is not one of semiconvection, since semiconvection does not normally occur to a significant extent in the portion of the MS band at issue $\left(\sim 2-8 M_{\odot}\right)$. Larger cores can be obtained by ad hoc variants on the Schwarzschild criterion, although there is no good theoretical basis for those that have been proposed so far (Eggleton 1983a). Recent improvements in the opacity (Rogers \& Iglesias 1992) have decreased the problem, but not resolved it. Although larger convective cores appear to be necessary above $\sim 2 M_{\odot}$, lower mass stars $\left(\sim 1.25 M_{\odot}\right)$ as in M67 (Morgan \& Eggleton 1978; Pols 1995, private communication) appear not to need larger convective cores. It is by no means obvious what physical process could achieve this.

(d) Convective heat transport. For most of a stellar interior it is not necessary to have a model of convection more definitive than the mixing-length theory (Böhm-Vitense 1958). But in the outer layers of red supergiants a better theory is necessary. The radii of such stars is therefore quite uncertain. In fact, such stars do not have a 'radius' in a very meaningful sense. Their outer layers probably consist of a small number of plumes rising and falling in a fairly chaotic way. However the uncertainty in their radii is probably conflated with (and perhaps related to) the uncertainty in the ML rate in (a), (a2) above.

(e) Bolometric correction. To convert from a theoretical HRD to an observed one, or vice versa requires rather detailed and reliable models of stellar atmospheres. This does not seem to have been achieved yet for cool stars. in particular those with atmospheric opacity dominated by molecular bands (and which coincidentally also have a temperature structure dominated by the uncertain process of convection). Now that it is possible with HST to measure even the lower MS of some globular clusters, the uncertainty in the bolometric correction in such late dwarfs is probably the main source of disagreement with theoretical models.

There are many other sources of uncertainty, but I believe the above five are the most relevant to single-star evolution. Given these uncertainties, I believe that the approximate (and very simple) formulae of Eggleton et al. (1989) are adequate for Pop I; but they will be improved in the near future. A comparison in the HRD of the results of SE computations and these approximate formulae is shown in Figure 1.

Even if single-star evolution were well understood, binary evolution is 
much more uncertain, and not just because there is a larger space of initial parameters to explore. All of the following kinds of binary are likely to be important at some stage in the evolution of a globular (or Galactic) cluster: contact binaries (WUMas), RS CVns, Algols, symbiotic stars, Ba or $\mathrm{CH}$ stars, cataclysmic variables (CVs), high and low-mass X-ray binaries (HMXBs, LMXBs), millisecond binary pulsars (MSBPs) and double degenerates (DDs). All have problems over and above the single-star problems described in the previous Section. Some of these problems are the following: (i) ML is probably enhanced by the presence of a close companion, to a largely unknown extent (Eggleton \& Tout 1989).

(ii) AML, which can take place even with negligible $M L$, is difficult to quantify. Although GR gives a definite rate, which could be important in some CVs and DDs, magnetic braking with tidal friction (Verbunt \& Zwaan 1981) is quite uncertain, and may be dominant in most of the above types of binaries (WUMas, RS CVns, Algols, CVs, LMXBs, MSPBs) which have short periods and cool companions.

(iii) Common-envelope evolution (Paczyński 1976) is a very important process, but its outcome can only be modeled very crudely.

The above three processes, and several others which are uncertain but perhaps less so, can affect the evolution of binaries qualitatively, and not just quantitatively. The same initial binary might, with slightly different assumptions, become (Eggleton 1983b) either a CV (with period usually $\lesssim 0.5 \mathrm{~d}$ ) or a Ba star (with period usually $\gtrsim 400 \mathrm{~d}$ ). For the present, we have to be content with crude recipes for many stages of binary evolution.

Let us suppose, for the sake of argument, that the evolution of single stars, and also of interacting binaries with circular orbits, can be modeled to a sufficient accuracy for inclusion in an $\mathrm{N}$-body code. It will be necessary to modify the standard equations of motion

$$
m_{\mathrm{i}} \ddot{\mathbf{r}}_{\mathrm{i}}=\mathbf{F}_{\mathrm{i}}^{(\mathrm{Gr})} \equiv-\sum_{\mathrm{j} \neq \mathrm{i}} \frac{G m_{\mathrm{i}} m_{\mathrm{j}} \mathbf{r}_{\mathrm{ij}}}{r_{\mathrm{ij}}^{3}} \quad, \quad \mathbf{r}_{\mathrm{ij}} \equiv \mathbf{r}_{\mathrm{i}}-\mathbf{r}_{\mathrm{j}},
$$

for the following reasons. Firstly, we need a term for tidal friction (TF), which will ensure, in those binaries where at least one star grows large enough to interact with its companion, that the orbit is gradually circularised. Neither primordial binaries, nor those that are formed later by exchange reactions, by three-body interaction, or by tidal capture, will be circular to start with, but they will cicularise on a timescale that, empirically, appears to be fairly short compared with the nuclear timescale, provided that at least one component has a radius comparable to the separation of the components at periastron. Secondly, we need a term which operates during the mass-transfer phase (RLOF) that can be expected to occur not long after the circularisation of the orbit by TF. This term must 
conserve angular momentum in a binary which is undergoing mass transfer with no net mass loss. The second correction is necessary because equation (1), as applied just to a binary, but to a binary whose components possibly vary in mass, implies that the angular momentum per unit reduced mass is a constant; whereas in a binary undergoing conservative RLOF it is the total angular momentum that is constant. In other words, equation (1) with varying mass supposes that mass which leaves one star carries away the same specific momentum as the star it leaves, and if it arrives on the other star then it adds a different amount of momentum, to wit the specific momentum of the gainer rather than the donor.

I believe the following dynamical equation is probably the simplest that encompasses both requirements:

$$
m_{\mathrm{i}}(t) \ddot{\mathbf{r}}_{\mathrm{i}}+\dot{m}_{\mathrm{iT}}\left(\dot{\mathbf{r}}_{\mathrm{i}}-\mathbf{V}\right)=\mathbf{F}_{\mathrm{i}}^{(\mathrm{Gr})}+\mathbf{F}_{\mathrm{i}}^{(\mathrm{TF})}, \quad i=1,2
$$

where

$$
\begin{gathered}
\mathbf{V} \equiv \frac{m_{1} \dot{\mathbf{r}}_{1}+m_{2} \dot{\mathbf{r}}_{2}}{m_{1}+m_{2}}, \\
\dot{m}_{1}=\dot{m}_{1 \mathrm{~W}}+\dot{m}_{1 \mathrm{~T}}, \quad \dot{m}_{2}=\dot{m}_{2 \mathrm{~W}}+\dot{m}_{2 \mathrm{~T}}, \quad \dot{m}_{2 \mathrm{~T}}=-\dot{m}_{1 \mathrm{~T}},
\end{gathered}
$$

suffix $W$ referring to wind which leaves the star and goes to infinity, and suffix $T$ referring to the mass which is transferred from one star to the other. Finally,

$$
\mathbf{F}_{\mathrm{i}}^{(\mathrm{TF})} \equiv-\sum_{\mathrm{i} \neq \mathrm{j}} \frac{\lambda_{\mathrm{i}} R_{\mathrm{i}}^{8}+\lambda_{\mathrm{j}} R_{\mathrm{j}}^{8}}{r_{\mathrm{ij}}^{8}} \frac{\mathbf{r}_{\mathrm{ij}} \mathbf{r}_{\mathrm{ij}} \cdot \dot{\mathbf{r}}_{\mathrm{ij}}}{r_{\mathrm{ij}}^{2}},
$$

where $R_{\mathrm{i}}$ is the radius of the $i$-th star, and the $\lambda$ 's are TF coefficients. The $\lambda$ 's are of course dependent on the internal physics of the stars, but can probably be reasonably approximated on dimensional grounds as stellar mass divided by a timescale which is short compared with the thermal timescale of the star. This TF term has been incorporated into a 3-body code by Dr L. G. Kiseleva, and is found to give interesting and useful results in the context of triple stars as well as binaries. It is the simplest term we can think of that

(i) is radial, and antisymmetric in $i, j$, so that it conserves linear and angular momentum;

(ii) opposes the radial component of velocity, and so goes to zero as the orbit becomes circular;

(iii) crudely depends on the ratio of stellar to orbital radius in the way determined by Zahn (1977) from a detailed physical analysis of TF.

Note that the above TF term is not intended as an accurate representation of the very complicated physics that occurs during a tidal capture process 
(Mardling, this Symposium), where possibly a great amount of kinetic energy is dissipated chaotically on a short timescale. It is only intended as a prescription for a gradual circularisation.

The mass transfer term in equation (2) has the property that if $\dot{m}_{1 \mathrm{~W}}=$ $0=\dot{m}_{2 \mathrm{~W}}$, so that all the mass lost by one star is gained by the other, then total angular momentum is conserved, and we get $P^{-1} \propto m_{1}^{3} m_{2}^{3}$, $a^{-1} \propto m_{1}^{2} m_{2}^{2}$, where $P$ and $a$ are the period and semimajor axis, the latter being the constant radius of a circular orbit by virtue of the TF term in the previous evolution. These are the normal results of conservative RLOF. If, on the other hand, $\dot{m}_{1 \mathrm{~T}}=0=\dot{m}_{2 \mathrm{~T}}$, so that there is only wind from one or both stars and no RLOF, then as usual the angular momentum per unit reduced mass is constant and so we get $P^{-1} \propto\left(m_{1}+m_{2}\right)^{2}$, $a^{-1} \propto m_{1}+m_{2}, e=$ const., as expected; though we should note, for wide orbits, that these equations for the expansion of an orbit in response to ML assume that the ML does not vary significantly during an orbit. If there is instaneous mass ejection as in an SN explosion, then equation (2) will still apply and will give the right results for that limit. Of course, it is implicit in such modeling of ML that the mass is lost isotropically (in the frame of the mass-losing star) at sufficiently high speed that it does not accumulate significantly within even the cluster, let alone the binary. It is not obvious to me how equation (2) might be further generalised to take into account the possibility of AML by magnetic braking, for example, although such a generalisation would seem to be necessary.

Note that in equation (3) the velocity $\mathbf{V}$ is obtained by summing only over the two stars that are transferring mass, and that because the two masses are variable $\mathbf{V}$ is not, as one might have supposed, the velocity of the CG of the binary. We can of course generalise so that we can write these equations down, with a rather more elaborate notation than I care to use here, for all $i$ rather than just $i=1,2$. However since the TF force is a short-range one it should only be necessary to include the nearest neighbour, and perhaps in the case of some hierarchical triples the next nearest neighbour. Note also that in equation (2) the rate of mass transfer $\dot{m}_{\mathrm{T}}$ can be considered as a fairly simple function of the ratio of stellar radius $R_{1}$ (if $* 1$ is the loser) to the Roche lobe radius of $* 1$, the latter being itself a fairly simple function of separation $a$ and mass ratio $m_{1} / m_{2}$. The rate is usually taken to be zero if the radius ratio is less than unity, and a rapidly increasing function if the ratio is greater than unity (Paczyński \& Sienkiewicz 1972). However, we cannot just use the stellar radius obtained from our simple SE subroutine, because the mass transfer itself affects the radius if it is comparable to or faster than the thermal timescale of the star. It is possible, however, that this can be allowed for with a very simple extra differential equation for the degree of Roche-lobe overfill (Whyte \& 
Eggleton 1985).

To sum up, we see that (i) back-of-the-envelope formulae exist for bulk stellar (Pop I) properties as functions of $m, t$. They are being upgraded for state-of-the-art internal physics, and generalised to a range of metallicities; (ii) evolution of interacting binaries is still quite problematic; and (iii) to accommodate mass loss by winds and mass transfer by RLOF, the N-body dynamical equations should be modified slightly. This includes adding a tidal friction term in order to circularise the orbits of really close binaries.

I am indebted to Drs S. J. Aarseth, Zh. Han, L. G. Kiseleva, O. R. Pols, K.-P. Schröder, and C. A. Tout for many helpful conversations.

\section{References}

Aarseth, S. J. (1995) in Binaries in Clusters, ed. Milone, E. F., ASP series, in press

Alexander, D. R. \& Ferguson, J. W. (1994) ApJ, 437, 879

Andersen, J. (1991) A\&A Rev., 3, 91

Bettweiser, E. \& Sugimoto, D. (1984) MN, 208, 493

Böhm-Vitense, E. (1958) ZsAp, 46, 108

Conti, P. S. (1982) in Mass Loss from Astronomical Objects, ed Gondhalekar, P. M., RAL 82-075, p45

Eggleton, P. P., Fitchett M. J. \& Tout C. A. (1989) ApJ, 347, 998

Eggleton, P. P. (1983a) MN, 204, 449

Eggleton, P. P. (1983b) in The Origin and Evolution of Cataclysmic Binaries, eds Livio, M. \& Shaviv, G., p239

Eggleton, P. P. \& Tout, C. A. (1989) Sp. Sc. Rev., 50, 165

Hachisu, I., Nakada, Y., Nomoto, K. \& Sugimoto, D. (1978) Prog. Theor. Phys., 60, 393

Han, Zh., Podsiadlowski, P. \& Eggleton, P. P. (1994) MN, 270, 121

Humphreys, R. M. \& Davidson, K. (1979) ApJ, 232, 409

Judge, P. G. \& Stencel, R. E. (1991) ApJ, 371, 357

Lynden-Bell, D. \& Eggleton, P. P. (1980) MN, 191, 483

Merryfield, W. J. (1995) ApJ, 444, 318

Meynet, G., Mermilliod, J.-C. \& Maeder, A. (1993) A\&AS, 98, 477

Morgan, J. G. \& Eggleton, P. P. (1978) MN, 182, 219

Paczyński, B. (1976) in IAU Symp. 73, Structure and Evolution of Close Binary Systems, eds Eggleton, P., Mitton, S. \& Whelan, J., p75

Paczyński, B. \& Sienkiewicz, R. (1972) AA, 22, 73

Paczyński, B. \& Ziółkowski, J. (1968) Acta Astr., 18, 255

Pols, O. R., Tout, C. A., Eggleton, P. P. \& Han, Zh. (1995) MN, 274, 964

Popper, D. M. (1980) ARAA, 18, 115

Rogers, F. J. \& Iglesias, C. A. (1992) ApJS, 79, 507

Schaller, G., Schaerer, D., Meynet, G. \& Maeder, A. (1992) A\&AS, 96, 269

Schwarzschild, M. \& Härm, R. (1958) ApJ, 128, 348

Spruit, H, (1992) A\&A, 253, 131

Verbunt, F. \& Zwaan, C. (1981) A\&A, 100, L7

Weidemann, V., \& Koester, D. (1983) A\&A, 121, 77

Whyte, C. A. \& Eggleton, P. P. (1985) MN, 214, 357

Willis, A. J. (1985) in Interacting Binaries, eds Eggleton, P. P. \& Pringle, J. E. p103

Zahn, J.-P. (1977) A\&A, 57, 383 